\title{
Voltage-Gated Sodium Ion Channel Activation
}

National Cancer Institute

\section{Source}

National Cancer Institute. Voltage-Gated Sodium Ion Channel Activation. NCI Thesaurus. Code C88551.

A process that involves detection of alteration to the voltage across a membrane, and results in opening of the channel to become selectively permeable to sodium ions. This interaction plays a key role in conduction of nerve impulses throug hout the central and peripheral nervous systems. Deficiencies in this process may result in various channelopathies, featuring epilepsy, seizures and paralysis. 